\title{
FUNCTIONS OF BVC TYPE ${ }^{1,2}$
}

\author{
RICHARD E. HUGHS
}

Let $f$ be a measurable function defined on the closed unit square $Q=I \times I, I=[0,1]$. For every $x \in I$, let $f_{x}$ be the function of $y$ defined by $f_{x}(y)=f(x, y)$ and for every $y \in I$, let $f^{y}$ be the function of $x$ defined by $f^{y}(x)=f(x, y)$. Let $V\left(f_{x}\right)$ and $V\left(f^{y}\right)$ be the variations of $f_{x}$ and $f^{y}$ on $I$, respectively. The function $f$ is said to be BVC (of bounded variation in the sense of Tonelli and Cesari $[1 ; 2])$, if there are functions $g$ and $h$, which are equal to $f$ almost everywhere on $Q$, such that: $V\left(g_{x}\right)<\infty$ for almost all $x \in I, V\left(h^{y}\right)<\infty$ for almost all $y \in I$, $\int_{0}^{1} V\left(g_{x}\right) d x<\infty$ and $\int_{0}^{1} V\left(h^{y}\right) d y<\infty$. The purpose of this note is to show that if $f$ is BVC, then there is a single function $k$, which is equal to $f$ almost everywhere on $Q$, such that: $\int_{0}^{1} V\left(k_{x}\right) d x<\infty$ and $\int_{0}^{1} V\left(k^{y}\right) d y$ $<\infty$. This fact has already been established, [3], in the special case where $f$ is essentially linearly continuous.

Let $f$ be a function defined on $[a, b]$,

$$
P:\left[a=\beta_{0}<\beta_{1}<\cdots<\beta_{r-1}<\beta_{r}=b\right]
$$

be a partition of $[a, b]$, and define for $x \in\left(\beta_{m-1}, \beta_{m}\right],\left(x \in\left[\beta_{0}, \beta_{1}\right]\right.$ if $m=1), m=1,2, \cdots, r$, the functions:

$$
\begin{aligned}
\phi_{P}^{+}(f ; x) & =f(a)+\frac{1}{2} \sum_{i=1}^{m}\left\{\left[f\left(\beta_{i}\right)-f\left(\beta_{i-1}\right)\right]+\left|f\left(\beta_{i}\right)-f\left(\beta_{i-1}\right)\right|\right\}, \\
-\phi_{P} & (f ; x)=\frac{1}{2} \sum_{i=1}^{m}\left\{\left[f\left(\beta_{i}\right)-f\left(\beta_{i-1}\right)\right]-\left|f\left(\beta_{i}\right)-f\left(\beta_{i-1}\right)\right|\right\}
\end{aligned}
$$

and if $0 \leqq j<k \leqq r, v\left(f ; P ; \beta_{j}, \beta_{k}\right)=\sum_{i=j+1}^{k}\left|f\left(\beta_{i}\right)-f\left(\beta_{i-1}\right)\right|$. The functions $\phi_{P}^{+}, \phi_{P}$ are monotone, nondecreasing. The norm of $P$ is defined as $|P|=\max \left[\left|\beta_{i}-\beta_{i-1}\right|, i=1,2, \cdots, r\right]$.

Lemma. If $f$ is a $B V$ function on $[a, b]$ and $\left\{P_{n}\right\}$ is a sequence of partitions of $[a, b]$, each a refinement of its predecessor with limit ${ }_{n \rightarrow \infty}\left|P_{n}\right|$ $=0$, then $\operatorname{limit}_{n \rightarrow \infty} \phi_{P_{n}}^{+}(f ; x)$ and $\operatorname{limit}_{n \rightarrow \infty} \phi_{P_{n}}(f ; x)$ exist at all points of $[a, b]$. If these limits are designated by $\phi^{+}$and $\phi^{-}$respectively, then $f=\phi^{+}-\phi^{-}$at all points of continuity of $f$ and $V\left(\phi^{+-} \phi^{-}\right) \leqq V(f)$. 1960.

Presented to the Society, November, 26, 1960; received by the editors November 12,

1 This paper is a portion of a thesis, directed by Professor Casper Goffman, to be submitted to Purdue University in partial fulfillment of the requirements for the degree of Doctor of Philosophy.

2 This research was sponsored by the National Science Foundation, grant number G-5867. 
Proof. Let $\left\{P_{n}\right\}$ be a sequence of partitions of $[a, b]$, where each is a refinement of its predecessor and $\operatorname{limit}_{n \rightarrow \infty}\left|P_{n}\right|=0$. Let $x$ be any number in $[a, b]$ and let $\left(\alpha_{n}, \beta_{n}\right]$ be that subinterval of $P_{n}$ which contains $x,\left(\left[\alpha_{n}, \beta_{n}\right]\right.$ if $\left.x=0\right)$.

Now,

$$
\begin{aligned}
\dot{\phi}_{P_{n}}^{+}(f ; x) & =f(a)+\left[f\left(\beta_{n}\right)-f(a)+v\left(f ; P_{n} ; a, \beta_{n}\right)\right] / 2 \\
& =f(a) / 2+f\left(\beta_{n}\right) / 2+v\left(f ; P_{n} ; a, \alpha_{n}\right) / 2+\left|f\left(\alpha_{n}\right)-f\left(\beta_{n}\right)\right| / 2
\end{aligned}
$$

and

$$
\overline{\phi_{P_{n}}}(f ; x)=f(a) / 2-f\left(\beta_{n}\right) / 2+v\left(f ; P_{n} ; a, \alpha_{n}\right) / 2+\left|f\left(\beta_{n}\right)-f\left(\alpha_{n}\right)\right| / 2 .
$$

But, $\left\{\alpha_{n}\right\}$ is a monotone, nondecreasing sequence, $\left\{\beta_{n}\right\}$ is a monotone, nonincreasing sequence and $\operatorname{limit}_{n \rightarrow \infty} \alpha_{n}=\operatorname{limit}_{n \rightarrow \infty} \beta_{n}=x$. Since $f$ is BV, it follows that $\operatorname{limit}_{n \rightarrow \infty} f\left(\beta_{n}\right)$ and $\operatorname{limit}_{n \rightarrow \infty}\left|f\left(\beta_{n}\right)-f\left(\alpha_{n}\right)\right|$ exist, and $\operatorname{limit}_{n \rightarrow \infty} v\left(f ; P_{n} ; a, \alpha_{n}\right)$ exists because $\left\{v\left(f ; P_{n} ; a, \alpha_{n}\right)\right\}$ is a monotone, nondecreasing sequence which is bounded above by the variation of $f$ on $[a, x]$. Thus, $\operatorname{limit}_{n \rightarrow \infty} \phi_{P_{n}}^{+}(f ; x)$ and $\operatorname{limit}_{n \rightarrow \infty} \phi_{P_{n}}(f ; x)$ exist for all $x \in[a, b]$. Clearly, $\operatorname{limit}_{n \rightarrow \infty}\left[\phi_{P_{n}}^{+}(f ; x)-\phi_{P_{n}}(f ; x)\right]$ $=\phi^{+}(f ; x)-\phi^{-}(f ; x)$ which equals $f(x+)$ if $x \neq \beta_{n}$ for any $n=1,2,3, \cdots$, and $f(x)$ if $x=\beta_{n}$ for some $n=1,2,3, \cdots$, which is just $f(x)$ at all $x$ which are points of continuity of $f$. In either case, it is clear that $V\left(\phi^{+}-\phi^{-}\right) \leqq V(f)$.

THEOREM. Let $f$ be a measurable function on the square, $Q$, which is $B V C$. Then, there is a single function, $k$, equal to $f$ almost everywhere on $Q$, for which the sections $k_{x}$ and $k^{y}$ are $B V$ almost everywhere in $x$ and $y$ respectively and $\int_{0}^{1} V\left(k_{x}\right) d x<\infty$ and $\int_{0}^{1} V\left(k^{y}\right) d y<\infty$.

PRoof. Since $f$ is BVC on $Q$, there are functions $g$ and $h$, equal to $f$ almost everywhere, such that their sections $g_{x}$ and $h^{y}$ are BV almost everywhere in $x$ and $y$ respectively and for which $\int_{0}^{1} V\left(g_{x}\right) d x<\infty$ and $\int_{0}^{1} V\left(h^{y}\right) d y<\infty$.

Let $\left\{P_{n}\right\}$ be a sequence of partitions of $I$, each one a refinement of the previous one, with the following properties: $\operatorname{limit}_{n \rightarrow \infty}\left|P_{n}\right|=0$; if $P_{n}:\left[0=\beta_{0}^{n} \leqq \beta_{1}^{n}<\beta_{2}^{n}<\cdots<\beta_{r_{n}}^{n} \leqq \beta_{r_{n}+1}^{n}=1\right]$, then $\beta_{1}^{n}, \cdots, \beta_{r_{n}}^{n}$ are such that $G(x)=g\left(x, \beta_{i}^{n}\right)$ is summable for $n=1,2,3, \cdots$ and $i=1,2, \cdots, r_{n} ; \operatorname{limit}_{n \rightarrow \infty} \beta_{1}^{n}=0$; $\operatorname{limit}_{n \rightarrow \infty} \beta_{r_{n}}^{n}=1$; and $\beta$ is any element of $P_{n}$ for all $n=1,2,3, \cdots$ for which $g(x, \beta)$ is summable in $x$.

For each $n=1,2,3, \cdots$, define, on the interval $\left[\beta_{1}^{n}, \beta_{r_{n}}^{n}\right] \subset I$, functions $\phi_{P_{n}}^{+}\left(g_{x}, y\right)$ and $\phi_{P_{n}}\left(g_{x}, y\right)$ exactly as described prior to the lemma where $g_{x}$ is BV. Then, one defines: 


$$
g_{P_{n}}^{+}(x, y)= \begin{cases}\phi_{P_{n}}^{+}\left(g_{x}, y\right) & \text { if } y \in\left[\beta_{1}^{n}, \beta_{r_{n}}^{n}\right] \text { and } g_{x} \text { is } \mathrm{BV}, \\ \phi_{P_{n}}^{+}\left(g_{x}, \beta_{1}^{n}\right) & \text { if } 0 \leqq y \leqq \beta_{1}^{n} \text { and } g_{x} \text { is } \mathrm{BV}, \\ \phi_{P_{n}}^{+}\left(g_{x}, \beta_{r_{n}}^{n}\right) & \text { if } \beta_{r_{n}}^{n} \leqq y \leqq 1 \text { and } g_{x} \text { is } \mathrm{BV}, \\ g(x, y) & \text { if } g_{x} \text { is not } \mathrm{BV} .\end{cases}
$$

Similarly, define $g_{P_{n}}(x, y)$ if $g_{x}$ is BV and let it be 0 if $g_{x}$ is not BV.

Consider now, $g_{P_{n}}^{+}$and $g_{P_{n}}$. If $\beta_{j-1}^{n}<y \leqq \beta_{j}^{n}, 2 \leqq j \leqq r_{n}$, one has that

$$
\begin{aligned}
g_{P_{n}}^{+}(x, y)= & g_{x}\left(\beta_{1}^{n}\right) \\
& +\frac{1}{2} \sum_{i=2}^{j}\left\{\left[g_{x}\left(\beta_{i}^{n}\right)-g_{x}\left(\beta_{i-1}^{n}\right)\right]+\left|g_{x}\left(\beta_{i}^{n}\right)-g_{x}\left(\beta_{i-1}^{n}\right)\right|\right\}
\end{aligned}
$$

if $g_{x}$ is BV, i.e. for almost all $x$. But, since $g\left(x, \beta_{i}^{n}\right)=g_{x}\left(\beta_{i}^{n}\right)$, $i=1,2, \cdots, r_{n}$ is a summable function of $x$ for $n=1,2,3, \cdots$, it follows that $g_{P_{n}}^{+}(x, y)$ is a measurable, and in fact summable, function of $(x, y) \in Q$. Similarly $g \bar{P}_{n}(x, y)$ is a measurable and summable function on $Q$. Although the form of $g_{P_{n}}^{+}$and $g_{P_{n}}$ is not identical to that of $\phi_{P_{n}}^{+}$and $\phi_{P_{n}}^{-}$in the previous lemma, the only essential distinction is that instead of a $g_{x}(0)$ term, there is a $g_{x}\left(\beta_{1}^{n}\right)$ term appearing, where $g_{x}$ is BV. Hence, by the lemma, limit ${ }_{n \rightarrow \infty} g_{P_{n}}^{+}(x, y)$ and limit ${ }_{n \rightarrow \infty} g_{P_{n}}(x, y)$ exist for all $(x, y) \in Q$. Let $g^{+}$and $g^{-}$be these limits, respectively, then $g^{+}$and $g^{-}$are measurable since each is a limit of a sequence of measurable functions.

Suppose $0<\alpha<1$ and $0 \leqq x \leqq 1$. Then, there is $N>0$ so that $n>N$ implies $P_{n}$ is such that $\beta_{1}^{n} \leqq \alpha \leqq \beta_{r_{n}}^{n}$. Hence, for $n>N, g_{P_{n}}^{+}(x, \alpha)$ $-g_{P_{n}}(x, \alpha)$ is equal to $\phi_{P_{n}}^{+}\left(g_{x}, \alpha\right)-\phi_{P_{n}}\left(g_{x}, \alpha\right)$ if $g_{x}$ is BV, and $g(x, \alpha)$ if $g_{x}$ is not BV, and thus, by the lemma, one has that the limit, $g^{+}(x, \alpha)-g^{-}(x, \alpha)$, is either $g(x, \alpha)$ or $g(x, \alpha+)$ depending upon whether $\alpha=\beta_{j}^{n}$ for some $j, n=1,2,3, \cdots$ and $g_{x}$ is BV. Thus, $g^{+}(x, y)-g^{-}(x, y)=g(x, y)$ at all points $(x, y) \in Q$ such that either $g_{x}$ is not BV or $g_{x}$ is BV and continuous at $y$. Since a BV function can be discontinuous at no more than a countable number of points, if $S$ is the set for which $g^{+}-g^{-}$differs from $g, S$ is measurable since $g^{+}$, $g^{-}$and $g$ are measurable, $m\left(S_{x}\right)=0$ for all $x$, where $S_{x}=\{y:(x, y) \in S\}$ and $m_{2}(S)=\int_{0}^{1} m\left(S_{x}\right) d x$, thus $m_{2}(S)=0$. Hence, $g^{+-} g^{-}$equals $g$ almost everywhere on $Q$ and $g_{x}^{+}$and $g_{x}^{-}$are monotone for almost all $x \in I$. Also, $V\left(g_{x}^{+}-g_{x}^{-}\right) \leqq V\left(g_{x}\right)$. 
It is clear from the definition of $g^{+}$and $g^{-}$, where $\beta \in P_{n}$, $n=1,2,3, \cdots$, that since $|g(x, y)| \leqq|g(x, \beta)|+V\left(g_{x}\right)$, it follows that both $\left|g^{+}(x, y)\right|$ and $\left|g^{-}(x, y)\right|$ are bounded by $|g(x, \beta)|+2 V\left(g_{x}\right)$ and since $V\left(g_{x}\right)$ and $g(x, \beta)$ are both summable on $Q, g^{+}$and $g^{-}$are also summable on $Q$.

Let $\left(g^{+}\right)^{s}$ and $\left(g^{-}\right)^{s}$ be the integral means of $g^{+}$and $g^{-}$, i.e., $\left(g^{+}\right)^{8}(x, y)$ $=s^{-2} \int_{y}^{y+s} \int_{x}^{x+s} g^{+}(u, v) d u d v, 0 \leqq x, y \leqq 1, g^{+}$is continued periodically and similarly for $g^{-}$. It is clear that $\left(g^{+}\right)_{x}^{s}$ and $\left(g^{-}\right)_{x}^{s}$ are monotone for all $x$ since $g_{x}^{+}$and $g_{x}^{-}$are monotone for almost all $x$ and it is well known that $\left(g^{+}\right)^{8}$ and $\left(g^{-}\right)^{8}$ are continuous and converge almost everywhere on $Q$ to $g^{+}$and $g^{-}$as $s$ goes to zero. Thus, $k^{+}=\lim \sup _{s \rightarrow 0}\left(g^{+}\right)^{s}$ and $k^{-}=\lim \sup _{s \rightarrow 0}\left(g^{-}\right)^{s}$ have the same properties as $g$ relative to $f$; i.e., $k=k^{+}-k^{-}$is equal almost everywhere to $f, k_{x}$ is BV for almost all $x, k_{x}^{+}$and $k_{x}^{-}$are monotone and $V\left(k_{x}\right) \leqq V\left(g_{x}\right)$ for almost all $x$. Thus, $\int_{0}^{1} V\left(k_{x}\right) d x<\infty$.

By exactly the same argument with $h$, the same function $k$ is obtained due to the symmetry of the integral means with respect to $x$ and $y$. Thus, there is a single function, $k$, equal almost everywhere to $f$, for which $k_{x}$ and $k^{y}$ are BV for almost all $x$ and $y$ respectively, and

$$
\int_{0}^{1} V\left(k_{x}\right) d x<\infty \text { and } \int_{0}^{1} V\left(k^{y}\right) d y<\infty .
$$

\section{REFERENCES}

1. L. Cesari, Sulle funzioni a variazione limitata, Ann. Scuola Norm Sup. Pisa (2) vol. 5 (1936) pp. 299-313.

2. K. Krickeberg, Distributionen, Funktionen beschränkter Variation und Lebesguescher Inhalt nichtparametrischer Flächen, Ann. Mat. Pura Appl., IV, vol. 44 (1957) pp. 105-133.

3. C. Goffman, Non-parametric surfaces given by linearly continuous functions, Acta Math. vol. 103 (1960) pp. 269-291.

Purdue University 